\title{
Paediatrics in the accident and emergency department
}

\author{
B M Phillips, W J Robson
}

When the National Health Service began in 1948 many hospitals, large and small, had casualty departments. These were usually run by experienced and dedicated nursing staff in often inadequate accommodation. Self taught clinical assistants were in overall charge but had inexperienced, often poorly supervised junior medical staff. The departments treated patients, including children, with a wide range of trivial or serious illness and injury.

In 1962, the Platt committee recommended that the renamed 'accident and emergency' departments be under the direction of orthopaedic surgeons. ${ }^{1}$ However, this solution was not generally successful and in 1971 the Joint Consultants Committee under Sir John Bruce suggested that a new type of specialist be created, namely consultant in accident and emergency medicine. ${ }^{2}$ There are now over 200 such specialists in England and Wales.

\section{Range of paediatric problems in accident and emergency medicine}

Little national or regional data are regularly gathered about children's accident and emergency attendances. Most districts record the number of child attenders under the age of 16 years, but no other details. Our knowledge of children's problems in accident and emergency is therefore mainly based on small local studies.

It is estimated that between $20 \%$ and $25 \%$ of the child population is treated annually at accident and emergency departments amounting to between 2 and 2.5 million children a year in England and Wales. ${ }^{3}$ In a typical health district with a large district general hospital whose accident and emergency department has 60000 new attenders per year, 20-30\% (15 000 to 20000 ) will be children.

The largest group of child attenders are those with trauma. Most of these have minor injuries. The commonest minor trauma is a head or facial injury in a child under 5 years old (B M Phillips, unpublished observations). Lacerations, simple fractures and sprains, animal bites, ingestions and foreign bodies, and burns and scalds, comprise most of the remainder of the minor trauma problems.

It is estimated that at least $15 \%$ of child attenders have medical conditions. These range from the seriously ill child with convulsions, collapse, or respiratory difficulty to patients who have conditions which could be treated by their general practitioner. In this latter group most are children with the common acute illnesses of childhood, especially respiratory and gastrointestinal infections. A minority have long standing conditions that have caused sudden concern, for example, recurrent abdominal pain or headaches. Children under 5 years and especially those under 2 years predominate in the medical attenders.

A small percentage of patients have nontraumatic surgical diseases such as irritable hip, Perthes' disease, appendicitis, hernia, etc. Some children, usually adolescents, attend with symptoms related to drugs, alcohol, or solvent abuse or after self harm.

Areas of special concern in child attenders at accident and emergency departments MAJOR TRAUMA

Trauma is the leading cause of death in children over the age of 1 year in the developed world. In England and Wales each year approximately 700 children die and about 10000 are permanently disabled as the result of accidents. ${ }^{4}$

In 1988, the Royal College of Surgeons of England's working party on the management of patients with major injuries reported that of 1000 deaths in all age groups from trauma (which were assessed retrospectively), 20\% were deemed to have been preventable if optimal treatment had been provided after the accident. ${ }^{5}$ Although the report does not make clear how children in the study fared compared with adults, it is likely that many deaths in childhood caused by trauma are preventable. A similar study in the United States considered that 53 out of 100 consecutive paediatric trauma deaths were potentially preventable with optimal treatment. ${ }^{6}$ A study of deaths in children with head injury from Newcastle identified $42 \%$ of 255 deaths as being possibly or probably avoidable with better treatment after arrival in hospital. ${ }^{7}$

The poor outcome probably arises from the relative inexperience and lack of training of staff in most hospitals when it comes to treating the severely injured child. Although trauma is the commonest cause of death in childhood, individual staff are likely to treat only one or two such patients a year. However, severely injured children urgently need the skills of trained and experienced accident and emergency staff, anaesthetists, and surgeons. The child requires rapid stabilisation with airway, ventilatory, and circulatory support and transfer to a unit experienced in paediatric trauma manage- 
ment. Paediatricians will recognise parallels here with the needs of the very small or very sick neonate.

The Royal College of Surgeons working party recommend the setting up of American style trauma centres in each region. In such a centre, specialist surgeons, anaesthetists, and operating theatres would always be available for the treatment of seriously injured patients. The working party recognised that severely injured children require paediatric facilities including paediatric surgeons, anaesthetists, and intensive therapy. It recommended that, where feasible, a major paediatric facility be part of the hospital containing the trauma centre. However, the working party considered it appropriate that injured children be transferred from the trauma centre to the children's hospital if the two were not on the same site. The Department of Health has recently funded a pilot centre to study the feasibility of trauma centres in the UK.

THE SERIOUSLY ILL CHILD

Early recognition and prompt, effective treatment of the seriously ill child in the accident and emergency department is vital, especially when the children's department and therefore the paediatric team is on a different site. These aims can be achieved only when the senior house officer in the accident and emergency department has in-post paediatric training and where trained and experienced middle grade staff are readily available.

\section{CHILDREN BROUGHT IN DEAD}

Children who have been the victims of a fatal accident or who have been found suddenly and unexpectedly dead are usually brought to the nearest accident and emergency department. Infants under 1 year old make up the majority of the latter group. Sensitive, caring, and supportive management of the family at this time will have a profound and prolonged positive influence on the response of the family to the death in the long term. Doctors, nurses, and social workers must be trained in these skills.

\section{CHILD ABUSE}

The problem of child abuse runs through the whole range of paediatric conditions treated in an accident and emergency department. Guidelines for recognition and management are necessary for this often complex situation. Clear lines of referral to senior paediatric staff should be agreed for children who present to the department with worrying injuries. However, the assessment of the child and his or her circumstances by the paediatrician and social worker is not best undertaken in the accident and emergency department unless there is a specially designated and staffed area for this purpose. The privacy and confidentiality required in these circumstances cannot always be maintained in a busy department.

\section{INAPPROPRIATE ATTENDERS}

Management of the inappropriate attender is a concern in all accident and emergency departments. An inappropriate attender is usually regarded as one whose condition is neither a recent accident nor an emergency and who would normally seek medical advice from his general practitioner. Many accident and emergency departments have a policy of referring such patients back to their general practitioner for treatment but this policy is not applied to children. ${ }^{8}$

In an unpublished study in a paediatric accident and emergency department, parents said it was not the unavailability of general practitioners that prompted them to bring their child to the paediatric accident and emergency department but their perception that the child's illness was an emergency and their belief that superior skills lay within the children's hospital (B M Phillips, unpublished observations). The accident and emergency department can be regarded as a 'safety net' where parents may obtain primary health care for their children if, for whatever reason, they have failed to obtain it elsewhere. In the inner cities there is a higher proportion of such child attenders compared with rural or suburban areas.

\section{Paediatric accident and emergency departments}

The joint statement on children's attendances at accident and emergency departments produced in 1988 by the British Paediatric Association, the British Association of Paediatric Surgeons, and the Casualty Surgeons Association (now the British Association for Accident and Emergency Medicine) recommends that paediatric accident and emergency medicine should develop as a specialty with the aim of establishing consultant posts in paediatric accident and emergency. medicine in children's hospitals. ${ }^{3}$ The statement recommends that there should be at least one paediatric accident and emergency unit in each region or large urban area based on a children's hospital or in a large accident and emergency unit within a hospital associated with a university department of child health.

There are at present five consultant led paediatric accident and emergency departments in England and Wales. In addition, there are two in Scotland, one in Northern Ireland, and two in the Republic of Ireland. These are all based on children's hospitals. In addition there are a few accident and emergency departments in children's hospitals with paediatric consultant supervision. Paediatric accident and emergency departments provide a service for the surrounding population and also attract a small number of additional 'medical' paediatric cases from further afield so that medical attenders comprise approximately $25 \%$ of the workload of these departments. ${ }^{3}$

The rationale for the existence of paediatric accident and emergency departments is:

- As a teaching and training resource for medical, nursing, and other staff.

- For research into the epidemiology and pathology of acute illness and injury.

- For the development of management strategies for acutely ill and injured children. 
CASUAL ATTENDERS IN PAEDIATRIC UNITS

In addition to identifiable paediatric accident and emergency units, some paediatric units operate an 'open door' policy for self referred patients, both old and new, to be treated in the unit's admissions department. This practice is only safe if resuscitation facilities and skills are available.

\section{Proposals for the future development of} paediatrics in district accident and emergency services

Several of the following proposals have already been put forward in the joint statement of the British Paediatric Association, the British Association of Paediatric Surgeons, and the Casualty Surgeons Association ${ }^{3}$ and in the quality review produced by the National Association for the Welfare of Children in Hospital. $^{9}$

\section{PHYSICAL FACILITIES}

It is proposed that:

- The accident and emergency department should be on the same site as the paediatric inpatient services so that experienced paediatric help will be readily available.

- The department should be clearly visible to families seeking treatment and should be well signposted.

- It should have a separate play and waiting area for children of sufficient size to cater for the average peak demand, not forgetting that many children are accompanied by siblings, therefore suitable toys and books for different ages groups should be provided.

- Consulting rooms should be big enough to accommodate the child, parents, and any siblings who have had to be brought in the emergency situation.

- There should be a separate children's treatment room suitably decorated and equipped.

- Children's waiting, play, and treatment areas should form a recognisably separate but contiguous subunit in the main department.

- There should be a private room for distressed parents or for private interviews with parents.

- There should be child size as well as adult toilet facilities and a nappy changing area.

- There should be a private area for mothers who are breast feeding.

- Enclosed areas are provided: they are more appropriate for children as they prevent children wandering. Sound attenuation is useful in both directions.

- Children's resuscitation facilities should not be separate from the main resuscitation area or there may not be sufficient staff to man both areas-however, one resuscitation bed should be for children with paediatric equipment and drug dosage protocols easily accessible.

MEDICAL STAFF TRAINING

Newly appointed senior house officers should have in-post training, which includes cardiopulmonary resuscitation in childhood, the initial management of the seriously ill or injured child, management of common paediatric problems, and recognition of child abuse.

They should be able to ventilate a child by bag and mask for a short period and should be trained in techniques of venous access including intraosseous infusion.

In-service training should continue on a regular basis. Attendance at an advanced cardiac or advanced trauma life support course would be very useful. There are plans to hold advanced paediatric life support courses in the UK similar to those in Canada and the United States.

\section{CONSULTANT TRAINING}

At present the Specialty Advisory Committee (SAC) on higher medical training requires only a minimum of three months paediatric experience before accreditation as a consultant in accident and emergency medicine. However, we suggest that a year's paediatric experience would be preferable to most candidates for such posts. The experience might appropriately be gained in a six months' paediatric senior house officer post at a district general hospital with a further six months of experience in paediatric specialities in the registrar or senior registrar years. These specialties should include intensive care and anaesthetics. A post in a paediatric accident and emergency department would also give valuable experience. In some large accident and emergency departments in district general hospitals where more than one consultant is appointed, we would recommend that one of the consultants should have a special interest in paediatric accident and emergency medicine with some additional training.

There are a few consultant posts in paediatric accident and emergency medicine. These are based in children's hospitals. Such departments treat children with a wide range of paediatric problems, including those pertinent to the hospital's tertiary specialist departments. Training for such posts falls under the scrutiny of both the SAC in paediatrics and the SAC in accident and emergency medicine. Discussions continue about the detailed content of such training programmes.

\section{NURSING STAFF}

It is proposed that:

- There should be one registered sick children's nurse on each shift.

- All nursing staff should have training in paediatric basic life support, drug administration, and equipment used for children.

- Further training should be available for nurses who wish to undertake extended rolesfor example, plastering, suturing, intubation and intravenous access, and ordering $x$ rays.

- Some departments develop the role of a clinical nurse practitioner who could treat patients with minor illness or injury without medical supervision.

- A paediatric district nurse is available to provide follow up in the community: this may avoid some admissions to hospital.

- There is liaison with the health visitors on all child attenders under the age of 5 years. 
FUNCTIONING OF THE ACCIDENT AND EMERGENCY DEPARTMENT WITH REGARD TO CHILDREN

Triage

Many accident and emergency departments now use a system of triage to assess and rank patients according to their degree of urgency. This task is performed by experienced nursing sisters or charge nurses and involves assessing the degree of severity of the patient's need and giving first aid treatment such as analgesics or splintage. In some cases investigations such as radiographs can be ordered. In childhood particular note should be made of the fact that 'ambulant' cases, which in adults implies 'not seriously ill', in children refers to the attending adult and not the child. Seriously ill babies can be carried into the accident and emergency department and their parents may wait patiently in a non-urgent queue while the infant's condition becomes worse. All infants should have triage performed immediately they have entered the accident and emergency department.

\section{Play}

Play is the means by which young children learn and understand; it distracts them from the distress associated with hospital attendance when ill or injured. Procedures and treatments may be explained to children using the vehicle of play. We recommend that a play specialist is appointed in the accident and emergency department for busy times and that the advice of a play specialist is available to the nursing staff.

\section{Protocols}

The wide variety of clinical problems and the urgency with which some present, coupled with the inexperience of junior accident and emergency doctors, makes the use of protocols in accident and emergency of great value. A protocol is not a rigid set of rules but is used to provide structure to thought and action in an emergency situation. ${ }^{10}$ Agreed protocols for managing situations such as cardiorespiratory arrest, the multiply injured child, the unconscious child, and the abused child should be drawn up with advice from appropriate specialists.

\section{Records and computerisation}

Separate identification of child attenders should be routine in accident and emergency departments. In addition, children of special interest, that is those with medical or social problems, should be easily identifiable. Computerisation of accident and emergency records with additional data on the epidemiology of accidents is of immense use for audit, planning, research, liaison, and accident prevention.

\section{Referral and liaison}

The majority of patients attending are managed in the accident and emergency department and its follow up clinics. A substantial minority are referred to other departments for further management. These principally include paediatric medicine, orthopaedics, surgery, burns and plastic surgery, ear, nose, and throat surgery, and neurosurgery. Involvement of anaesthetists is particularly important in the management of patients requiring resuscitation from illness or major injury and in the transfer of severely ill or injured patients to other hospitals. Each department should have medical social workers readily available. Community liaison should be established with doctors in child health, health visitors, and district nurses.

\section{Discharge of patients}

When children are discharged from the accident and emergency department ideally their general practitioners should be informed of their attendances and subsequent treatment. In practice, without computerised records, the numbers of attenders are too great to make this feasible. It is useful to give parents written instructions (including those for non-English speaking families) and leaflets should be available for parents' information in, for example: (i) care of wounds, (ii) plaster of Paris, (iii) observations after a head injury, (iv) management of gastroenteritis, (v) the feverish child, and (vi) the convulsing child.

\section{Audit}

Many different aspects of audit are relevant in the accident and emergency department. These include efficiency of the service, the appropriateness of clinical care, and referrals to other departments. Internal audit among the staff of the accident and emergency department and audit with other departments in the hospital are of equal value. It is also appropriate for departments in neighbouring districts to hold joint audit meetings.

\section{The role of the paediatrician}

The joint statement on children's attendances at accident and emergency departments recommends that all departments should have a consultant paediatrician with responsibility for liaison with the consultant in accident and emergency medicine, concerning the general arrangements for children. ${ }^{3}$ This can be achieved in several ways: (i) teaching sessions for accident and emergency staff, (ii) a weekly paediatric clinic in the accident and emergency department, (iii) joint audit meetings, and (iv) agreed protocols.

\section{National liaison}

The newly established British Paediatric Accident and Emergency Group (BPAEG) is affiliated to the British Paediatric Association and draws members from paediatrics and from accident and emergency medicine. Links like this and the Royal College of Nursing's Accident and Emergency Forum allow doctors and nurses from different disciplines who are involved in the management of acutely ill or injured children to work together. We propose that consideration should be given to a national committee for 
children's emergency services. Members could be drawn from paediatrics, accident and emergency medicine and allied specialties, nursing groups, ambulance services, and child accident prevention groups. The remit of such a committee would be similar to that of the Standing Committee on Emergency Medical Services for Children of the American Academy of Pediatrics-that is, responsibility for developing national standards of emergency care for children.

1 Standing Medical Advisory Committee, chairman Sir Harry Platt. Accident and emergency services. London: HMSO, 1962 .

2 Joint Consultants Committee, chairman Sir John Bruce.
Report of a joint working party. London: British Medical Association, 1971.

3 British Paediatric Association, British Association of Paediatric Surgeons, Casualty Surgeons Association. Foint statement on children's attendances at accident and emergency ment on children's attendances at accident and emergency
departments. London: British Paediatric Association, 1988.

4 Child Accident Prevention Trust. Basic principles of child Child Accident Prevention Trust. Basic principles of child
accident prevention. A guide to action. London: Child accident prevention. A guide to
Accident Prevention Trust, 1989.

5 Royal College of Surgeons of England. Report of the working party on the management of patients with major injuries. London: Royal College of Surgeons of England, 1988.

6 Ramenofsky ML, Luterman A, Quindlen E, et al. Maximum survival in pediatric trauma; the ideal system. $\mathcal{F}$ Trauma 1984;24:818-23.

7 Sharples PM, et al. Avoidable factors contributing to death of children with head injury. $B M F$ 1990;300:87-91.

8 Carew-McColl M. Gently adjusting open doors. Arch Emerg Med 1990;7:59-60.

9 National Association for the Welfare of Children in Hospital. Setting standards for children in health care. The NAWCH quality review 1989. London: National Association for the quality review 1989. London: National
Welfare of Children in Hospital, 1989.

10 Ludwig S, Selbst S. A child-oriented emergency medical services system. Curr Probl Pediatr 1990;20:149.

\section{Persistent proteinuria}

The Japanese seem to put an extraordinary amount of effort into screening their children. Not only do they screen for neuroblastoma (see Archivist 1991:1007) but they also test every school child's urine once a year between the ages of 6 and 15. In this way they have found a lot of children with proteinuria. Most of them had transient or intermittent proteinuria, orthostatic proteinuria, or proteinuria associated with haematuria. Between 1976 and 1989, 62 children had renal biopsies performed at Kobe University Hospital because of isolated non-postural proteinuria ( 1 to $4+$ on Albustix) lasting for six months or more. Fifty three had biopsy specimens adequate for examination by light microscopy, immunofluorescence, and electron microscopy and these form the basis of a recent report (Norishige Yoshikawa and colleagues, Fournal of Pediatrics 1991;119:375-9).

Forty two of the 53 children were discovered through the school screening programme. The biopsy specimens of 28 children (group 1) showed 'minimal change' (that is, they were normal or showed slight mesangial proliferation) and those of the remaining 25 (group 2) showed significant pathological changes. These were: focal segmental glomerulosclerosis (FSGS) $(n=15)$, IgA nephropathy $(n=4)$, diffuse mesangial proliferative glomerulonephritis without $\operatorname{IgA}$ deposition $(n=3)$, and membranous glomerulonephritis $(n=3)$. Two children with FSGS had had 'minimal change' biopsy appearances two years earlier. There were no clinical pointers at presentation to distinguish those with significant pathology. At follow up for an average of six years seven children $(28 \%)$ in group 2, all with FSGS, developed chronic renal failure. None in group 1 did so. Three out of 10 children with severe proteinuria and 10 of $\mathbf{4 3}$ with mild proteinuria went on to chronic renal failure. Children with persistent proteinuria should be followed up. A renal biopsy will give a better idea about prognosis but otherwise seems unlikely to affect management very much at present.

The routine annual testing of schoolchildren for proteinuria seems to have a greater potential for causing upset than for producing benefit. In Kobe with a population near to that of Scotland such testing detected about three children a year with persistent isolated proteinuria and presumably many more with transient or orthostatic proteinuria. In Finland $11 \%$ of nearly 9000 schoolchildren were found to have proteinuria without haematuria but in none was it persistent and none had evidence of significant renal pathology. ${ }^{1}$ In America routine urine testing in hospital has been deprecated (see Archivist 1991:266). The national psychology of screening programmes seems a subject worthy of study.

\section{ARCHIVIST}

